\title{
Facile formation of phosphono-acridanes via chloroacridines
}

\author{
Venu Srinivas and K. C. Kumara Swamy* \\ School of Chemistry, University of Hyderabad, Hyderabad-500046, A. P., India \\ E-mail: kckssc@yahoo.com, kckssc@uohyd.ernet.in
}

\begin{abstract}
Novel bis-phosphonates bearing an acridane moiety are readily synthesized by the simple reaction of appropriate 9-chloroacridines with phosphites of the type $(\mathrm{RO})_{2} \mathrm{P}(\mathrm{O}) \mathrm{H}$. The by-product acridones could be converted back to the chloroacridines by treating them with thionyl chloride, and thus the effective yield is high. The structures of three bis-phosphonates are confirmed by X-Ray crystallography.
\end{abstract}

Keywords: Bis-phosphonates, acridine, acridones, phosphonylation, X-ray structure

\section{Introduction}

Acridine derivatives have numerous applications ${ }^{1}$ that include biological fluorescent/ chemiluminiscent probes $^{2,3}$ as well as anti-tumor, ${ }^{4}$ anti-bacterial, ${ }^{5}$ anti-HIV, ${ }^{6}$ antimalarial, ${ }^{7}$ and DNA-binding agents. ${ }^{8} \quad$ Amsacrine [N-(4-(acridin-9-ylamino)-3-methoxyphenyl)-methanesulfonamide, 1] is a drug used in acute lymphoblastic leukemia. Proflavine (3,6-acridinediamine, 2), used effectively during World War II for deep wound dressing, has been studied extensively as a novel RNA-targeted antiviral drug and as an intercalator in cancer treatments. It has been the CAS molecule of the week (September 2005). Recently, some mono-, bis-, and tetra-acridines have also shown in vitro anti-parasitic activity against Leishmania infantum (e.g., 3). ${ }^{9}$

We have been interested in organophosphonates, ${ }^{10}$ and in this context wanted to prepare phosphono-substituted acridines for possible future drug-related applications. As precursors, we chose 9-chloroacridines. Since the addition of P-H bond to aryl halides is an important reaction for the preparation of organophosphorus compounds, ${ }^{11}$ and bis-phosphonates are clinically the most important class of anti-resorptive agents available to treat diseases characterized by osteoclastmediated bone resorption such as osteoporosis, Paget's disease, and tumor-associated bone diseases, ${ }^{12}$ we became more interested in this aspect. The results of our studies are reported herein. 
<smiles>COc1cc(NS(C)(=O)=O)ccc1Nc1c2ccccc2nc2ccccc12</smiles>

1 (Amsacrine)<smiles>Nc1ccc2cc3ccc(N)cc3nc2c1</smiles>

2 (Proflavine)<smiles>[R]c1ccc2nc3ccc(OC)cc3c([R])c2c1</smiles>

3 (Antiproliferative)

\section{Results and Discussion}

Treatment of anthranilic acids with phosphorus oxychloride $\left(\mathrm{POCl}_{3}\right)$ at $130{ }^{\circ} \mathrm{C}$ for $2-4 \mathrm{~h}^{13}$ afforded the corresponding substituted 9-chloroacridines 4-7. Compounds 8, 9 were also synthesized in a similar manner. Among these, 6-9 are new. When these 9-chloroacridines 4-9 were treated with the cyclic phosphite 10 under neat conditions at $90{ }^{\circ} \mathrm{C}$ for 2-4 h, the bis-phosphonates 11-16 were formed along with the corresponding acridones (Scheme 1). Although the yield of the compounds is only moderate (reaction mixture, single phosphonate product, yield in the reaction mixtures 50 $60 \%$; isolated yield of pure compounds 15-30\%) the formation of these compounds represents a new type of phosphonylation and is thus unprecedented.

We have made an attempt to increase the yield by changing the reaction conditions; however, we always ended up in having acridone (30-40\%) by-product, probably because of a competing $\mathrm{OH}$ to $-\mathrm{Cl}$ exchange between chloro-acridine and phosphite. However, this problem could be circumvented since the acridones formed were readily converted back to the chloroacridines by simple treatment with thionyl chloride. ${ }^{14}$ Isolation of 11-16 is also fairly straightforward.

An analogous reaction of $\mathbf{4}$ with diethyl phosphite afforded the corresponding bis-phosphonate 17. ${ }^{15}$ Only one phosphonate product is generally formed. Considering that the by-product acridone could be converted quantitatively into the corresponding chloroacridines, the effective yield is very good. Use of different solvents (THF and toluene) did not alter the yield significantly. Employing more than two mole equivalents of phosphite $\mathbf{1 0}$ did not significantly alter the yields, and even the use of less than one mole equivalent of $\mathbf{1 0}$ led mostly to the bis-phosphonate as the only solid phosphorus product $\left({ }^{31} \mathrm{P}\right.$ NMR). However, by using a lower stoiochiometry of phosphite $\mathbf{1 0}$ in its reaction with chloro-acridine $\mathbf{5}$, the mono-phosphonate $\mathbf{1 8}$ could be isolated.<smiles>[R]c1ccc2nc3ccccc3c(Cl)c2c1</smiles><smiles>Cc1c(Cl)ccc2c(Cl)c3ccccc3nc12</smiles>

8<smiles>Clc1c2ccccc2nc2c1ccc1ccccc12</smiles>

9 


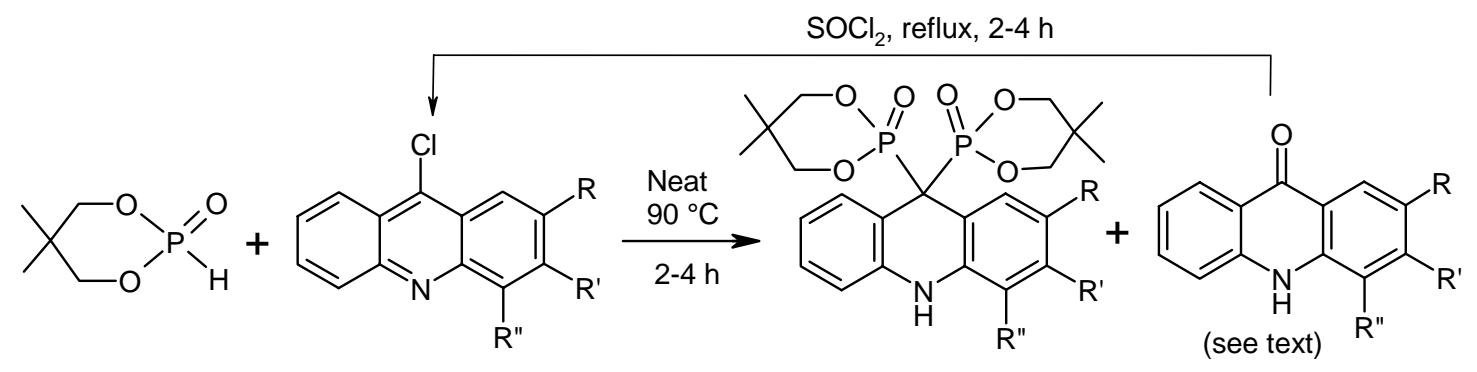

10

4-9

$\mathrm{R}=\mathrm{R}^{\prime}=\mathrm{R}{ }^{\prime}=\mathrm{H}(\mathbf{1 1}, \mathrm{X}$-ray $)$

$\mathrm{R}=\mathrm{Br}, \mathrm{R}^{\prime}=\mathrm{R}{ }^{\prime}=\mathrm{H}$ (12)

$\mathrm{R}=\mathrm{Me}, \mathrm{R}^{\prime}=\mathrm{R}$ " = H (13)

$\mathrm{R}=\mathrm{NO}_{2}, \mathrm{R}^{\prime}=\mathrm{R} "=\mathrm{H}$ (14, X-ray)

$\mathrm{R}=\mathrm{H}, \mathrm{R}$ ' = Cl, R" = Me (15)

$\mathrm{R}=\mathrm{H}, \mathrm{R}, \mathrm{R}^{\prime}{ }^{\prime}=\mathrm{C}_{4} \mathrm{H}_{4}$

(part of benzene ring)(16)

\section{Scheme 1}

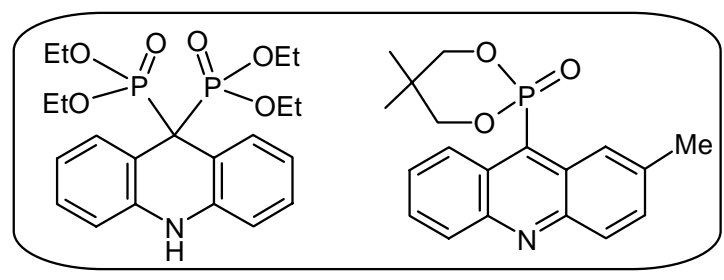

17 (X-ray)

18

A possible pathway for the formation is shown in Scheme 2. The monophosphonate of type I could undergo elimination of a molecule of $\mathrm{HCl}$ to afford the 9-phosphono-acridine II. Since the nitrogen site is basic, it can take up the available proton from the acid to lead to a species like III, in which the double bond attached to the phosphonate group is activated to accept a second phosphonate entity to lead to the bis-phosphonates 11-16. It is also possible that species I can simply reorganize to the amine salt III with chloride as the counter-ion, prior to the addition of the second molecule of 10. Evidence for the involvement of II comes from the fact that we could isolate the mono-phosphonate 18, albeit in modest yields. These reactions differ from the nucleophilic substitution reactions of these chloroacridines that lead to monosubstituted products. ${ }^{16}$ 

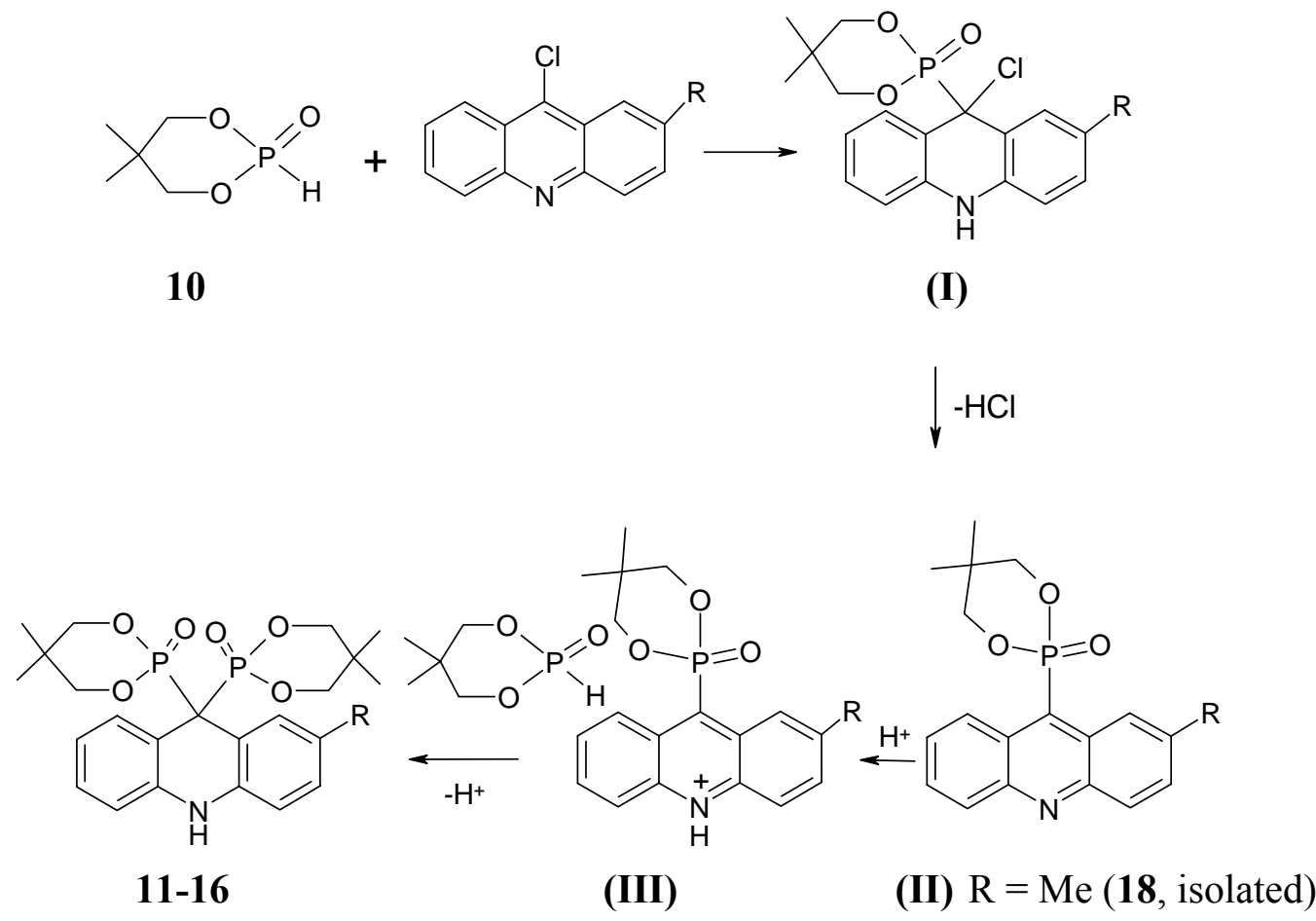

Scheme 2. Plausible reaction mechanism for the formation of bis-phosphonate.

\section{A brief discussion of the $\mathrm{X}$-ray structures of $11 . \mathrm{CH}_{3} \mathrm{CN}, 14 . \mathrm{OC}_{4} \mathrm{H}_{8}$ and 17}

The ORTEP drawings of $\mathbf{1 1} . \mathrm{CH}_{3} \mathrm{CN}, \mathbf{1 4} . \mathrm{OC}_{4} \mathrm{H}_{8}$ and $\mathbf{1 7}$ are shown in Figure 1. The $\mathrm{P}-\mathrm{C}$ distances are in the expected range for $\mathrm{P}-\mathrm{C}$ single bonds ${ }^{10 \mathrm{a}-\mathrm{c}, 17}$ although marginal variations are found in the three structures $(11,14$ and 17). There is clear-cut hydrogen- bonding interaction between the $\mathrm{NH}$ of the acridane residue and one of the $\mathrm{P}=\mathrm{O}$ groups of the phosphonate group in all the three compounds. This is exhibited by the formation of dimeric units in $11 . \mathrm{CH}_{3} \mathrm{CN}$ and $14 . \mathrm{OC}_{4} \mathrm{H}_{8}$. However, in the ethyl compound $\mathbf{1 7}$ the preference is in favor of a hydrogen-bonded chain rather than dimer formation. Interestingly, compound 11. $\mathrm{CH}_{3} \mathrm{CN}$ crystallizes in the trigonal space group R-3 which appears to be a manifestation of additional C-H... O hydrogen bonding interactions between the three protons of the solvent acetonitrile and an oxygen atom of 1,3,2dioxaphosphorinane of the ring as shown in Figure 2. We have observed before the involvement of acetonitrile hydrogen atoms in $\mathrm{C}-\mathrm{H}$...O interactions. ${ }^{18}$ 

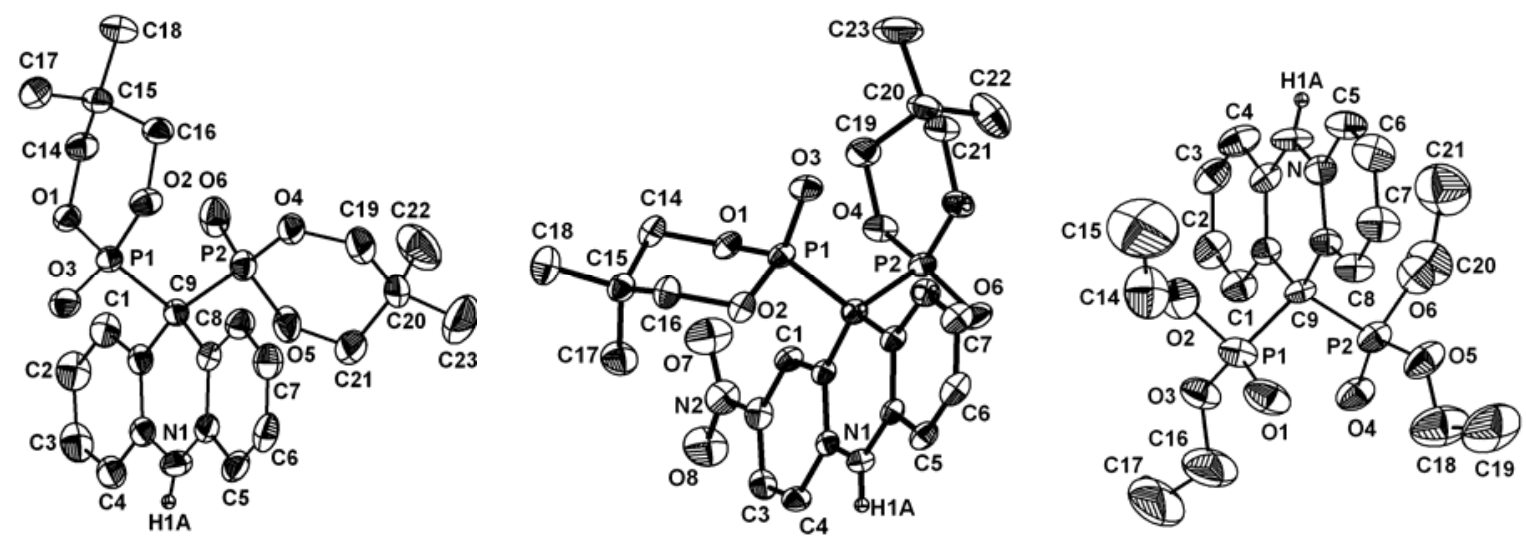

Figure 1. ORTEP diagrams of compounds 11. $\mathrm{CH}_{3} \mathrm{CN}$ (left, solvent molecule not shown), 14. $\mathrm{OC}_{4} \mathrm{H}_{8}$ (middle, solvent molecule not shown) and 17 (right). Selected P-C distances $(\AA)$ : Compound 11. $\mathrm{CH}_{3} \mathrm{CN}$ : $\mathrm{P}(1)-\mathrm{C}(9)$ 1.851(5), $\mathrm{P}(2)-\mathrm{C}(9)$ 1.853(5). Compound 14. $\mathrm{OC}_{4} \mathrm{H}_{8}$ : $\mathrm{P}(1)-\mathrm{C}(9)$ 1.829(5), $\mathrm{P}(2)-\mathrm{C}(9)$ 1.848(5). Compound 17: $\mathrm{P}(1)-\mathrm{C}(9)$ 1.864(5), $\mathrm{P}(2)-\mathrm{C}(9)$ 1.849(5).

(a)

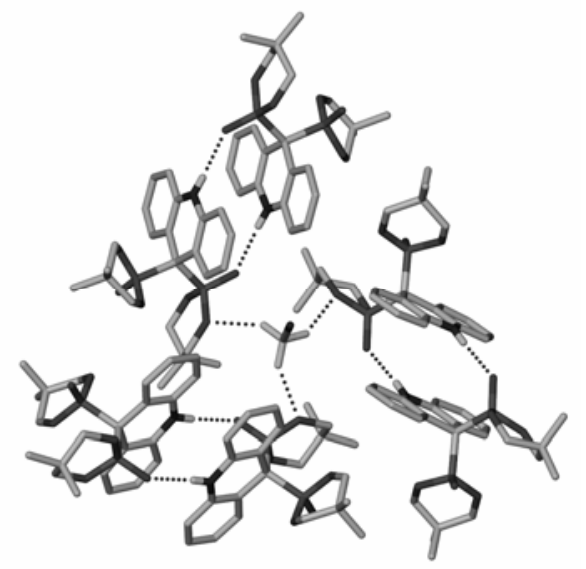

(b)

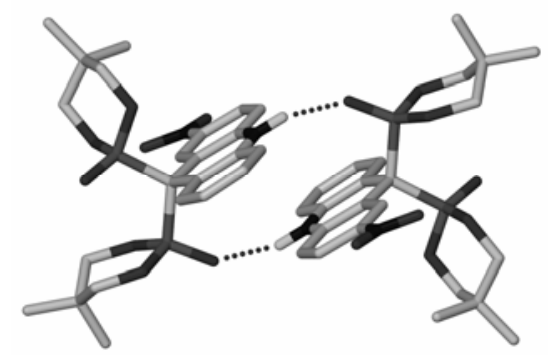

(c)

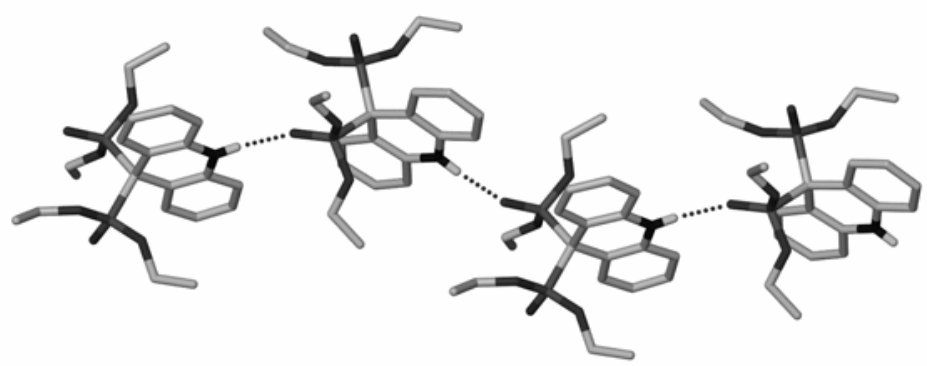

Figure 2. Drawings showing hydrogen- bonding interactions in (a) $11 . \mathrm{CH}_{3} \mathrm{CN}$, (b) $14 . \mathrm{OC}_{4} \mathrm{H}_{8}$, and (c) 17. Hydrogen- bonding interactions: Compound 11. $\mathrm{CH}_{3} \mathrm{CN}\left(\AA,{ }^{\circ}\right)$ : $\mathrm{N}(1)-\mathrm{H}(1 \mathrm{~A}) \ldots \mathrm{O}\left(3^{\prime}\right)$ 0.77(4), 2.18(4), 2.952(7), 176(4); C(25)-H(25A)...O(2) 1.07(7), 2.41(8), 3.361(8), 147(6). Symm. Equiv.: 5/3-x, 4/3-y, 1/3-z. Compound 14. $\mathrm{OC}_{4} \mathrm{H}_{8} \mathrm{~N}(1)-\mathrm{H}(1 \mathrm{~A}) \ldots \mathrm{O}(6$ ') 0.86, 1.99, 2.820(6), 162.5. Symm. Equiv.: 2-x, 1-y, -z. Compound 17 N-H(1A)...O(4') 0.78(5), 2.18(5), 2.910(6), 155(5). Symm. Equiv.: 2-x, 0.5+y, -z. 
The cytotoxicity $\left(\mathrm{IC}_{50}\right.$ in $\mu \mathrm{M}$ ) for compound $\mathbf{1 3}$ with cell lines Hep $\mathrm{G} 2$ and HeLa was found to be 150 and 79 , respectively. ${ }^{19}$ These data suggest that our compounds are fairly active, and efforts are under way to obtain the corresponding diphosphonic acids and check their activity.

\section{Conclusions}

A novel and very simple synthesis of bis-phosphonate bearing an acridane component, via the reaction of chloro-acridines with phosphites of the type $(\mathrm{RO})_{2} \mathrm{P}(\mathrm{O}) \mathrm{H}$, has been discovered. Since the by-product acridones can be converted back readily to the chloroacridines the overall yield is quite good. The preliminary cytotoxicity data for $\mathbf{1 3}$ appears to be encouraging.

\section{Experimental Section}

General. Chemicals were procured from Aldrich or local manufacturers and were purified when required ${ }^{20}{ }^{1} \mathrm{H}-,{ }^{13} \mathrm{C}$ - and ${ }^{31} \mathrm{P}$ - NMR spectra $\left({ }^{1} \mathrm{H}-400 \mathrm{MHz},{ }^{13} \mathrm{C}-100 \mathrm{MHz}\right.$ and ${ }^{31} \mathrm{P}-162 \mathrm{MHz}$, respectively) were recorded using a BRUKER $400 \mathrm{MHz}$ spectrometer in $\mathrm{CDCl}_{3}$ (unless stated otherwise) with shifts referenced to $\mathrm{SiMe}_{4}(\delta=0)$ or $85 \% \mathrm{H}_{3} \mathrm{PO}_{4}(\delta=0)$. IR spectra were recorded on a JASCO FT-IR 5300 spectrophotometer. Melting points were determined by using a local hotstage melting point apparatus and are uncorrected. Elemental analyses were carried out on a PerkinElmer 240C CHN analyzer. Mass spectra were recorded using GCMS-QP2010 and LCMS-2010A instruments. $\mathrm{N}$-phenylanthranilic acid derivatives were prepared according to a reported procedure. ${ }^{21}$ The cyclic phosphite $\mathbf{1 0}$ was prepared by a method previously reported from our laboratory. ${ }^{22}$

\section{(a) Synthesis of the substituted 9-chloroacridines 4-9}

The appropriate anthranilic acid $(6.7 \mathrm{mmol})$ was dissolved in $\mathrm{POCl}_{3}(10 \mathrm{~mL})$ and heated under reflux for $4 \mathrm{~h}$. After cooling to room temperature, excess of $\mathrm{POCl}_{3}$ was distilled off, and the mixture was added very carefully with vigorous stirring to a mixture containing crushed ice, aq. ammonia $(10 \mathrm{~mL})$ and chloroform $(30 \mathrm{~mL})$, making sure that the solution was always basic. The aqueous layer was extracted with chloroform $(2 \times 30 \mathrm{~mL})$ and combined with the original organic layer, dried over anhyd. $\mathrm{CaCl}_{2}$. The solvent was removed to yield the crude product. Analytically pure samples were obtained after flash-chromatography. Compounds 4-5 are known. Data for the new compounds 6-9 are given below.

9-Chloro-2-bromo-acridine (6). Yield: $1.38 \mathrm{~g}, 71 \%$; mp 160-162 ${ }^{\circ} \mathrm{C}$; brown solid; IR (KBr) 3123, 3088, 2980, 1636, 1595, 1524, 1466, 1346, $754 \mathrm{~cm}^{-1}$; ${ }^{1} \mathrm{H}$ NMR (400 MHz, CDCl $) \delta 7.92$ (t, $1 \mathrm{H}, J$ $\sim 7.8 \mathrm{~Hz}, \operatorname{Ar}-H), 8.14-8.18$ (m, $2 \mathrm{H}, \mathrm{Ar}-H), 8.60(\mathrm{~d}, 1 \mathrm{H}, J=8.8 \mathrm{~Hz}, \mathrm{Ar}-\mathrm{H}), 8.76(\mathrm{~d}, 1 \mathrm{H}, J=1.5 \mathrm{~Hz}$, Ar-H), $8.93(\mathrm{~d}, 1 \mathrm{H}, J \sim 9.0 \mathrm{~Hz}, \operatorname{Ar}-H), 8.98(\mathrm{~d}, 1 \mathrm{H}, J \sim 9.0 \mathrm{~Hz}, \operatorname{Ar}-H) ;{ }^{13} \mathrm{C}$ NMR $(100 \mathrm{MHz}$, $\left.\mathrm{CDCl}_{3}\right) \delta 121.6,124.5,124.7,125.0,126.6,127.7,129.6,131.1,131.2,134.5,146.8,148.6$; LC-MS $\mathrm{m} / \mathrm{z}=294[\mathrm{M}+1]^{+}$. Anal. Calcd for $\mathrm{C}_{13} \mathrm{H}_{7} \mathrm{BrClN}$ : C, 53.37; H, 2.41; N, 4.79. Found: C, 53.41; H, $2.41 ; \mathrm{N}, 4.74 \%$. 
9-Chloro-2-nitro-acridine (7). Yield: $0.87 \mathrm{~g}, 50 \%$; mp $172-174{ }^{\circ} \mathrm{C}$; yellow solid; IR (KBr,) 3088, 2919, 2847, 1935, 1634, 1520, 1354, $764 \mathrm{~cm}^{-1}$; ${ }^{1} \mathrm{H}$ NMR (400 MHz, $\left.\mathrm{CDCl}_{3}\right) \delta 7.63$ (td, $1 \mathrm{H}, J \sim 8.0$ $\mathrm{Hz}, J \sim 2.0 \mathrm{~Hz}, \operatorname{Ar}-H), 7.75(\sim \mathrm{t}, 1 \mathrm{H}, J \sim 8.0 \mathrm{~Hz}, \operatorname{Ar}-H), 8.25$ (d, $1 \mathrm{H}, J \sim 8.8 \mathrm{~Hz}, \operatorname{Ar}-H), 8.33(\mathrm{~d}, 1$ $\mathrm{H}, J=9.5 \mathrm{~Hz}, \operatorname{Ar}-H), 8.47$ (d, $1 \mathrm{H}, J=8.8 \mathrm{~Hz}, \operatorname{Ar}-H), 8.48-8.52(\mathrm{~m}, 1 \mathrm{H}, \mathrm{Ar}-H), 9.41$ (d, $1 \mathrm{H}, J=$ $2.0 \mathrm{~Hz}, \mathrm{Ar}-\mathrm{H}) ;{ }^{13} \mathrm{C} \mathrm{NMR}\left(100 \mathrm{MHz}, \mathrm{CDCl}_{3}\right) \delta 122.8,123.7,125.1,128.5,130.0,131.9,133.1$, 145.1, 145.7, 149.2, 150.7; LC-MS m/z $=259[\mathrm{M}+1]^{+}$. Anal. Calcd for $\mathrm{C}_{13} \mathrm{H}_{7} \mathrm{ClN}_{2} \mathrm{O}_{2}: \mathrm{C}, 60.36 ; \mathrm{H}$, 2.73 ; N, 10.83. Found: C, 60.48; H, 2.69; N, 10.96\%.

3,9-Dichloro-4-methylacridine (8). Yield: $1.30 \mathrm{~g}$, 75\%; mp 150-152 ${ }^{\circ} \mathrm{C}$; light yellow solid; IR $\left(\mathrm{KBr}, \mathrm{cm}^{-1}\right) 2966,1601,1549,1520,1454,1395,1314,1009,752 ;{ }^{1} \mathrm{H}$ NMR $\left(400 \mathrm{MHz}, \mathrm{CDCl}_{3}\right) \delta$ $2.98\left(\mathrm{~s}, 3 \mathrm{H}, \mathrm{CH}_{3}\right), 7.53(\mathrm{~d}, 1 \mathrm{H}, J=9.3 \mathrm{~Hz}, \mathrm{Ar}-H), 7.62(\sim \mathrm{t}, 1 \mathrm{H}, J \sim 7.6 \mathrm{~Hz}, \mathrm{Ar}-H), 7.78(\sim \mathrm{t}, 1 \mathrm{H}, J$ 7.6 Hz, Ar-H), 8.18 (d, $1 \mathrm{H}, J=9.3 \mathrm{~Hz}, \operatorname{Ar}-H), 8.22$ (d, $1 \mathrm{H}, J=8.8 \mathrm{~Hz}, \operatorname{Ar}-H), 8.36(\mathrm{~d}, 1 \mathrm{H}, J=$ $8.8 \mathrm{~Hz}, \mathrm{Ar}-H) ;{ }^{13} \mathrm{C} \mathrm{NMR}\left(100 \mathrm{MHz}, \mathrm{CDCl}_{3}\right) \delta 15.0,122.7,123.0,123.8,124.5,127.1,128.5,130.4$, 130.5, 134.9, 135.5, 144.2, 148.3, 148.5. Anal. Calcd for $\mathrm{C}_{14} \mathrm{H}_{9} \mathrm{Cl}_{2} \mathrm{~N}$ : C, 64.15; H, 3.46; N, 5.34. Found: C, 64.22; H, 3.47; N, 5.24\%.

7-Chloro-benzo[c]acridine (9). Yield: $1.17 \mathrm{~g}, 60 \%$; mp 144-146 ${ }^{\circ} \mathrm{C}$; light yellow solid; IR ( $\mathrm{KBr}$, $\left.\mathrm{cm}^{-1}\right)$ 3036, 1624, 1557, 1493, 1470, 1393, 1304, 1238, 742; ${ }^{1} \mathrm{H}$ NMR (400 MHz, $\left.\mathrm{CDCl}_{3}\right) \delta 7.70$ $(\sim \mathrm{t}, 1 \mathrm{H}, J \sim 8.0 \mathrm{~Hz}, \mathrm{Ar}-H), 7.75-7.82(\mathrm{~m}, 3 \mathrm{H}, \mathrm{Ar}-H), 7.85-7.90(\mathrm{~m}, 2 \mathrm{H}, \mathrm{Ar}-H), 8.21(\mathrm{~d}, 1 \mathrm{H}, J=$ $9.3 \mathrm{~Hz}, \operatorname{Ar}-H), 8.40$ (d, $1 \mathrm{H}, J=8.6 \mathrm{~Hz}, \operatorname{Ar}-H), 8.46$ (d, $1 \mathrm{H}, J=8.6 \mathrm{~Hz}, \operatorname{Ar}-H), 9.50$ (d, $1 \mathrm{H}, J=7.9$ $\mathrm{Hz}, \mathrm{Ar}-\mathrm{H}) ;{ }^{13} \mathrm{C} \mathrm{NMR}\left(100 \mathrm{MHz}, \mathrm{CDCl}_{3}\right) \delta 121.8,122.7,124.5,124.9,125.7,127.0,127.7,128.0$, $129.1,129.5,130.1,130.2,131.4,133.6,140.1,147.6,147.9 ; \mathrm{LC}-\mathrm{MS} \mathrm{m} / \mathrm{z}=264[\mathrm{M}+1]^{+}$. Anal. Calcd for $\mathrm{C}_{17} \mathrm{H}_{10} \mathrm{ClN}$ : C, 77.42; H, 3.82; N, 5.31. Found: C, 77.41; H, 3.84; N, 5.53\%.

(b) Preparation of the 9,9-diphosphono-10-hydroacridanes (11-17) using 9-chloroacridines: Representative procedure for 11

In a $25 \mathrm{~mL}$ round bottom flask, 9-chloroacridine $(1.07 \mathrm{~g}, 5 \mathrm{mmol})$ and the phosphite 10 (1.50 g, 10 mmol) were heated at $90{ }^{\circ} \mathrm{C}$ under nitrogen for $4 \mathrm{~h}$. At this stage, only one phosphorus signal due to the product was observed. When there was no 9-chloroacridine left (TLC), ethyl acetate (20 $\mathrm{mL})$ was added to the reaction mixture followed by saturated sodium bicarbonate solution. The organic layer was separated and aqueous layer extracted thrice $(3 \times 10 \mathrm{~mL})$ with ethyl acetate. The combined organic part was washed with water $(5 \times 20 \mathrm{~mL})$, brine solution, dried over anhyd. $\mathrm{Na}_{2} \mathrm{SO}_{4}$ and concentrated to yield brown colored gummy solid. Pure 9,9-bis-(5,5-dimethyl-2-oxo$2 \lambda^{5}$-[1,3,2] dioxaphosphinan-2-yl)-9,10-dihydro-acridine (11) was isolated by column chromatography (ethyl acetate: hexane 4:1) as a white solid. Other compounds 12-17 were prepared similarly. The reaction mixture showed a single phosphonate product in most cases, in ca 50-60\% yield $\left({ }^{1} \mathrm{H}\right.$ NMR). We could also recover the corresponding acridone formed as a byproduct, and convert it back to chloroacridine in nearly quantitative yields by treatment with thionyl chloride.

9,9-Bis-(5,5-Dimethyl-2-oxo-2- $\boldsymbol{\lambda}^{\mathbf{5}}$-[1,3,2]dioxaphosphinan-2-yl) $\quad$ 9,10-dihydro-acridine (11). Yield (by NMR; isolated) 55\%, $0.50 \mathrm{~g}(20 \%)$; mp 248-250 ${ }^{\circ} \mathrm{C}$; white solid; IR (KBr) 3266, 2965, 1613, 1530, 1491, 1344, 1250, $1064 \mathrm{~cm}^{-1} ;{ }^{1} \mathrm{H}$ NMR (400 MHz, $\left.\mathrm{CDCl}_{3}\right) \delta 0.71\left(\mathrm{~s}, 6 \mathrm{H}, 2 \mathrm{CH}_{3}\right), 1.22$ (s, $\left.6 \mathrm{H}, 2 \mathrm{CH}_{3}\right), 3.91-3.97\left(\mathrm{~m}, 4 \mathrm{H}, 2 \mathrm{OCH}_{2}\right), 4.15-4.17\left(\mathrm{~m}, 4 \mathrm{H}, 2 \mathrm{OCH}_{2}\right), 6.03$ (d, $2 \mathrm{H}, J=7.6 \mathrm{~Hz}$, Ar-H), 6.64 ( t, $2 \mathrm{H}, J \sim 7.6 \mathrm{~Hz}, \mathrm{Ar}-H), 6.71$ ( t, $2 \mathrm{H}, J \sim 7.6 \mathrm{~Hz}, \mathrm{Ar}-H), 7.80$ (d, $2 \mathrm{H}, J=7.6 \mathrm{~Hz}$, 
Ar- $H), 7.96(\mathrm{~s}, 1 \mathrm{H}, \mathrm{NH}) ;{ }^{13} \mathrm{C}$ NMR $\left(100 \mathrm{MHz}, \mathrm{CDCl}_{3}\right) \delta 20.7,22.5,32.4$ and $32.5\left(2 \mathrm{~d}, J_{(\mathrm{P}-\mathrm{C})} \sim 4.4\right.$ $\left.\mathrm{Hz}, \mathrm{CMe}_{2}\right), 51.8\left(\mathrm{t}, J_{(\mathrm{P}-\mathrm{C})}=132.1 \mathrm{~Hz}, \mathrm{P}-\mathrm{C}-\mathrm{P}\right), 78.3,110.0$ and $110.1\left(2 \mathrm{~d}, J_{(\mathrm{P}-\mathrm{C})} \sim 7.2 \mathrm{~Hz}\right), 115.0$, $118.4,129.4,131.7,139.0$ and $139.2\left(2 \mathrm{~d}, J_{(\mathrm{P}-\mathrm{C})} \sim 5.5 \mathrm{~Hz}\right) ;{ }^{31} \mathrm{P}$ NMR $\left(162 \mathrm{MHz}, \mathrm{CDCl}_{3}\right) \delta 8.0(\mathrm{~s})$; LC-MS $m / z=478[\mathrm{M}+1]^{+}$; Anal. Calcd for $\mathrm{C}_{23} \mathrm{H}_{29} \mathrm{NO}_{6} \mathrm{P}_{2}: \mathrm{C}, 57.86 ; \mathrm{H}, 6.12 ; \mathrm{N}, 2.93$. Found: $\mathrm{C}$, $58.04 ; \mathrm{H}, 6.10 ; \mathrm{N}, 2.84 \%$. This compound was crystallized from acetonitrile $(2 \mathrm{~mL})$. An X-ray structure analysis was performed on this compound (left diagram in Figure 1).

2-Bromo-9,9-bis-(5,5-dimethyl-2-oxo-2 $\lambda^{5}$-[1,3,2] dioxaphosphinan-2-yl) 9,10-dihydro-acridine (12). Yield [by NMR; isolated] 58\%, $0.72 \mathrm{~g}(26 \%)$; mp 268-270 ${ }^{\circ} \mathrm{C}$; white solid; IR $\left(\mathrm{KBr}, \mathrm{cm}^{-1}\right)$ 3254, 3177, 2967, 1615, 1489, 1244, 1069, 1028; ${ }^{1} \mathrm{H}$ NMR $\left(400 \mathrm{MHz}, \mathrm{CDCl}_{3}\right) \delta 0.75$ (s, $6 \mathrm{H}, 2$ $\left.\mathrm{CH}_{3}\right), 1.26$ (s, $\left.6 \mathrm{H}, 2 \mathrm{CH}_{3}\right), 4.01$ (not resolved, $\left.\left.4 \mathrm{H}, 2 \mathrm{x} \mathrm{OCH}\right)_{2}\right), 4.22$ and $4.24\left(\sim 2 \mathrm{~d}, 4 \mathrm{H}, J_{(\mathrm{H}-\mathrm{H})} \sim J_{(\mathrm{P}-}\right.$ н) $\left.7.1 \mathrm{~Hz}, 2 \mathrm{OCH}_{2}\right), 5.92-5.98(\mathrm{~m}, 2 \mathrm{H}, \mathrm{Ar}-\mathrm{H}), 6.68-6.77(\mathrm{~m}, 3 \mathrm{H}, \mathrm{Ar}-\mathrm{H}), 7.78(\mathrm{~d}, 1 \mathrm{H}, J=6.6 \mathrm{~Hz}$, Ar- $H), 7.86(\mathrm{~s}, 1 \mathrm{H}), 8.39(\mathrm{~s}, 1 \mathrm{H}) .{ }^{13} \mathrm{C}$ NMR $\left(100 \mathrm{MHz}, \mathrm{CDCl}_{3}\right) \delta 20.6,22.4,32.4$ and $32.5(2 \mathrm{~d}$, $\left.J_{(\mathrm{P}-\mathrm{C})} \sim 4.0 \mathrm{~Hz}, C \mathrm{Me}_{2}\right), 51.6\left(\mathrm{t}, J_{(\mathrm{P}-\mathrm{C})}=131.0 \mathrm{~Hz}, \mathrm{P}-\mathrm{C}-\mathrm{P}\right), 78.5$ and $78.6\left(2 \mathrm{~d},{ }^{2} J_{(\mathrm{P}-\mathrm{C})} \sim 11.0 \mathrm{~Hz}\right.$, $\left.\mathrm{OCH}_{2}\right), 109.5$ and $109.6\left(2 \mathrm{~d},{ }^{2} J_{(\mathrm{P}-\mathrm{C})} \sim 8.0 \mathrm{~Hz}\right), 109.9,111.9,115.2,116.3,118.8,129.6,131.5$, 132.1, 134.0, 138.6, 138.8; ${ }^{31} \mathrm{P}$ NMR $\left(162 \mathrm{MHz}, \mathrm{CDCl}_{3}\right) \delta 7.3(\mathrm{~s}) ; \mathrm{LC}-\mathrm{MS} \mathrm{m} / \mathrm{z}=556[\mathrm{M}]^{+}$. Anal. Calcd for $\mathrm{C}_{23} \mathrm{H}_{28} \mathrm{BrNO}_{6} \mathrm{P}_{2}: \mathrm{C}, 49.66 ; \mathrm{H}, 5.07 ; \mathrm{N}, 2.52$. Found: $\mathrm{C}, 49.61 ; \mathrm{H}, 5.12 ; \mathrm{N}, 2.70 \%$.

9,9-Bis-(5,5-dimethyl-2-oxo-2 $\lambda^{5}$-[1,3,2]dioxaphosphinan-2-yl)-2-methyl-9,10-dihydro-acridine (13). Yield [by NMR; isolated] 52\%, $0.74 \mathrm{~g}(30 \%)$; mp 262-264 ${ }^{\circ} \mathrm{C}$; white solid; IR $\left(\mathrm{KBr}, \mathrm{cm}^{-1}\right)$ 3272, 2963, 2926, 1615, 1495, 1343, 1256, 1066. ${ }^{1} \mathrm{H}$ NMR $\left(400 \mathrm{MHz}, \mathrm{CDCl}_{3}\right) \delta 0.71$ (s, $6 \mathrm{H}, 2$ $\left.\mathrm{CH}_{3}\right), 1.22\left(\mathrm{~s}, 6 \mathrm{H}, 2 \mathrm{CH}_{3}\right), 2.25\left(\mathrm{~s}, 3 \mathrm{H}, \mathrm{CH}_{3}\right), 3.92-3.98\left(\mathrm{~m}, 4 \mathrm{H}, 2 \mathrm{OCH}_{2}\right), 4.13-4.16(\mathrm{~m}, 4 \mathrm{H}, 2$ $\left.\mathrm{OCH}_{2}\right), 6.00(\mathrm{~d}, 1 \mathrm{H}, J=7.6 \mathrm{~Hz}, \mathrm{Ar}-H), 6.05(\mathrm{~d}, 1 \mathrm{H}, J=7.9 \mathrm{~Hz}, \mathrm{Ar}-\mathrm{H}), 6.53(\mathrm{~d}, 1, \mathrm{H}, J=7.9 \mathrm{~Hz}$, Ar-H), $6.64(\sim \mathrm{t}, 1 \mathrm{H}, J \sim 7.6 \mathrm{~Hz}, \mathrm{Ar}-H), 6.72(\sim \mathrm{t}, 1 \mathrm{H}, J \sim 7.6 \mathrm{~Hz}, \mathrm{Ar}-H), 7.61(\mathrm{~s}, 1 \mathrm{H}), 7.82(\mathrm{~d}, 1 \mathrm{H}$, $J=7.6 \mathrm{~Hz}, \mathrm{Ar}-H), 7.86(\mathrm{~s}, 1 \mathrm{H}) .{ }^{13} \mathrm{C} \mathrm{NMR}\left(100 \mathrm{MHz}, \mathrm{CDCl}_{3}\right) \delta 20.7,21.1,22.4,32.4$ and $32.5(2 \mathrm{~d}$, $\left.J_{(\mathrm{P}-\mathrm{C})} \sim 4.2 \mathrm{~Hz}, C \mathrm{Ce}_{2}\right), 51.8\left(\mathrm{t}, J_{(\mathrm{P}-\mathrm{C})}=132.4 \mathrm{~Hz}, \mathrm{P}-\mathrm{C}-\mathrm{P}\right), 78.2,109.7$ and $109.8\left(2 \mathrm{~d}, J_{(\mathrm{P}-\mathrm{C})} \sim 9.2 \mathrm{~Hz}\right)$, 114.7 and $114.8\left(2 \mathrm{~d}, J_{(\mathrm{P}-\mathrm{C})} \sim 7.9 \mathrm{~Hz}\right), 118.2,127.3,129.2,130.1,131.9\left(\mathrm{t}, J_{(\mathrm{P}-\mathrm{C})}=3.6 \mathrm{~Hz}\right), 136.7$, 139.4; ${ }^{31} \mathrm{P}$ NMR $\left(162 \mathrm{MHz}, \mathrm{CDCl}_{3}\right) \delta 8.1(\mathrm{~s})$; LC-MS $m / z=492[\mathrm{M}+1]^{+}$. Anal. Calcd for $\mathrm{C}_{24} \mathrm{H}_{31} \mathrm{NO}_{6} \mathrm{P}_{2}: \mathrm{C}, 58.65 ; \mathrm{H}, 6.36 ; \mathrm{N}, 2.85$. Found : C, 58.64; H, 6.36; N, 2.86\%.

\section{9,9-Bis-(5,5-dimethyl-2-oxo-2 $\lambda^{5}$-[1,3,2] dioxaphosphinan-2-yl)-2-nitro-9,10-dihydro-acridine}

(14). Yield [by NMR; isolated] 50\%, $0.39 \mathrm{~g}(15 \%)$; mp 272-274 ${ }^{\circ} \mathrm{C}$; yellow solid; IR $\left(\mathrm{KBr}, \mathrm{cm}^{-1}\right)$ 2963, 2924, 2855, 1624, 1491, 1262, 1092, 1020, 800; ${ }^{1} \mathrm{H}$ NMR (400 MHz, $\left.\mathrm{CDCl}_{3}\right) \delta 0.76(\mathrm{~s}, 6 \mathrm{H}, 2$ $\mathrm{CH}_{3}$ ), 1.28 (s, $\left.6 \mathrm{H}, 2 \mathrm{CH}_{3}\right), 4.04-4.13\left(\mathrm{~m}, 4 \mathrm{H}, 2 \mathrm{OCH}_{2}\right), 4.24-4.28$ (not resolved, $4 \mathrm{H}, 2 \mathrm{OCH}_{2}$ ), 5.98 and 6.00 (2 closely spaced d, $2 \mathrm{H}, \mathrm{Ar}-\mathrm{H}), 6.71(\sim \mathrm{t}, 1 \mathrm{H}, J \sim 7.8 \mathrm{~Hz}, \mathrm{Ar}-\mathrm{H}), 6.82(\sim \mathrm{t}, 1 \mathrm{H}, J \sim$ $7.8 \mathrm{~Hz}, \mathrm{Ar}-H), 7.47$ (d, $1 \mathrm{H}, J=8.8 \mathrm{~Hz}, \mathrm{Ar}-H), 7.83(\mathrm{~d}, 1 \mathrm{H}, J=7.8 \mathrm{~Hz}, \mathrm{Ar}-H), 8.76(\mathrm{~d}, 1 \mathrm{H}, J=1.9$ $\mathrm{Hz}, \mathrm{Ar}-\mathrm{H}), 9.27(\mathrm{~s}, 1 \mathrm{H}, \mathrm{NH}) ;{ }^{13} \mathrm{C} \mathrm{NMR}\left(100 \mathrm{MHz}, \mathrm{CDCl}_{3}\right) \delta 20.6,22.2,32.6$ and $32.7\left(2 \mathrm{~d}, J_{(\mathrm{P}-\mathrm{C})} \sim\right.$ $\left.4.2 \mathrm{~Hz}, \mathrm{CMe}_{2}\right), 52.1$ (t, $\left.J_{(\mathrm{P}-\mathrm{C})} \sim 132.2 \mathrm{~Hz}, \mathrm{P}-\mathrm{C}-\mathrm{P}\right), 78.6,110.0$ and $110.7\left(2 \mathrm{~d}, J_{(\mathrm{P}-\mathrm{C})} \sim 8.0 \mathrm{~Hz}\right), 114.1$, $115.7,121.0,125.3,129.2,129.8,131.6,137.2\left(\mathrm{~d}, J_{(\mathrm{P}-\mathrm{C})} \sim 5.3 \mathrm{~Hz}\right), 139.0,144.8\left(\mathrm{~d}, J_{(\mathrm{P}-\mathrm{C})} \sim 4.3 \mathrm{~Hz}\right)$; ${ }^{31} \mathrm{P}$ NMR $\left(162 \mathrm{MHz}, \mathrm{CDCl}_{3}\right) \delta 7.1$ (s); $\mathrm{LC}-\mathrm{MS} m / z=522[\mathrm{M}]^{+}$. Anal. Calcd for $\mathrm{C}_{23} \mathrm{H}_{28} \mathrm{~N}_{2} \mathrm{O}_{8} \mathrm{P}_{2}: \mathrm{C}$, 52.88; H, 5.40; N, 5.36. Found: C, 52.79; H, 5.45; N, 5.45\%. This compound was crystallized from dichloromethane $(3 \mathrm{~mL})$ containing traces of THF. X-Ray structural analysis was performed on this sample (middle diagram in Figure 1). 
3-Chloro-9,9-bis-(5,5-dimethyl-2-oxo-2 $\lambda^{5}$-[1,3,2]dioxaphosphinan-2-yl)-4-methyl-9,10-dihydroacridine (15). Yield [by NMR; isolated] 54\%, $0.52 \mathrm{~g} \mathrm{(20 \% );} \mathrm{mp} \mathrm{280-282}{ }^{\circ} \mathrm{C}$; yellow solid; IR $\left(\mathrm{KBr}, \mathrm{cm}^{-1}\right) 3285,2965,1611,1470,1258,1061 ;{ }^{1} \mathrm{H} \mathrm{NMR}\left(400 \mathrm{MHz}, \mathrm{CDCl}_{3}\right) \delta 0.73(\mathrm{~s}, 6 \mathrm{H}, 2$ $\mathrm{CH}_{3}$ ), 1.17 (s, $6 \mathrm{H}, 2 \mathrm{CH}_{3}$ ), 1.90 (s, $3 \mathrm{H}, \mathrm{CH}_{3}$ ), 3.98-4.02 (s, not resolved, $4 \mathrm{H}, 2 \mathrm{OCH}_{2}$ ), 4.08-4.11 $\left(\mathrm{m}, 4 \mathrm{H}, 2 \mathrm{OCH}_{2}\right), 6.53(\mathrm{~d}, 1 \mathrm{H}, J=7.8 \mathrm{~Hz}, \mathrm{Ar}-H), 6.72-6.83(\mathrm{~m}, 3 \mathrm{H}, \mathrm{Ar}-H), 6.93(\mathrm{~s}, 1 \mathrm{H}, \mathrm{NH})$, $7.74(\mathrm{~d}, 1 \mathrm{H}, J=8.7 \mathrm{~Hz}, \operatorname{Ar}-H), 7.85(\mathrm{~d}, 1 \mathrm{H}, J=7.8 \mathrm{~Hz}, \mathrm{Ar}-H) ;{ }^{13} \mathrm{C}$ NMR $\left(100 \mathrm{MHz}, \mathrm{CDCl}_{3}\right) \delta$ 13.5, 20.8, 22.3, 32.4 and $32.5\left(2 \mathrm{~d}, J_{(\mathrm{P}-\mathrm{C})} \sim 3.1 \mathrm{~Hz}, C \mathrm{Me}_{2}\right), 52.0\left(\mathrm{t}, J_{(\mathrm{P}-\mathrm{C})}=132.0 \mathrm{~Hz}, \mathrm{P}-C-\mathrm{P}\right), 77.9$ and $78.0\left(2 \mathrm{~d}, J_{(\mathrm{P}-\mathrm{C})} \sim 4.0 \mathrm{~Hz}, \mathrm{OCH}_{2}\right), 109.4$ and $110.3\left(2 \mathrm{~d}, J_{(\mathrm{P}-\mathrm{C})} \sim 7.0 \mathrm{~Hz}\right), 115.5,119.4,119.8$, 120.0, 129.4, 130.5, 132.0, 135.2, 138.2, 138.4; ${ }^{31} \mathrm{P}$ NMR (162 MHz, $\left.\mathrm{CDCl}_{3}\right) \delta 8.0$ (s); LC-MS m/z $=525[\mathrm{M}-1]^{+}$. Anal. Calcd for $\mathrm{C}_{24} \mathrm{H}_{30} \mathrm{ClNO}_{6} \mathrm{P}_{2}: \mathrm{C}, 54.81 ; \mathrm{H}, 5.75 ; \mathrm{N}, 2.66$. Found: C, 54.88; H, $5.71 ; \mathrm{N}, 2.69 \%$.

7,7-Bis-(5,5-dimethyl-2-oxo-2 $\lambda^{\mathbf{5}}$-[1,3,2]dioxaphosphinan-2-yl)-7,12-dihydro-benzo-[c]-acridine (16). Yield [by NMR; isolated] 52\%, $0.26 \mathrm{~g}(15 \%)$; mp 298-300 ${ }^{\circ} \mathrm{C}$; yellow solid; IR $\left(\mathrm{KBr}, \mathrm{cm}^{-1}\right)$ 3295, 3061, 2963, 1607, 1535, 1495, 1414, 1262, 1061, 745; ${ }^{1} \mathrm{H}$ NMR (400 MHz, CDCl $)_{3} \delta .66(\mathrm{~s}$, $6 \mathrm{H}, 2 \mathrm{CH}_{3}$ ), $1.21\left(\mathrm{~s}, 6 \mathrm{H}, 2 \mathrm{CH}_{3}\right), 3.91-3.99\left(\mathrm{~m}, 4 \mathrm{H}, 2 \mathrm{OCH}_{2}\right), 4.13$ (not resolved, $4 \mathrm{H}, 2 \mathrm{OCH}_{2}$ ), 6.40-6.43 (m, $1 \mathrm{H}, \mathrm{Ar}-H), 6.53$ ( t, $2 \mathrm{H}, J \sim 7.2 \mathrm{~Hz}, \mathrm{Ar}-H), 7.09-7.15$ (m, $2 \mathrm{H}, \mathrm{Ar}-H)$, 7.24-7.27 (m, $1 \mathrm{H}, \operatorname{Ar}-H), 7.45$ (d, $1 \mathrm{H}, J=7.8 \mathrm{~Hz}, \operatorname{Ar}-H), 7.73-7.79$ (m, $2 \mathrm{H}, \operatorname{Ar}-H), 7.92$ (d, $1 \mathrm{H}, J=8.8 \mathrm{~Hz}, \operatorname{Ar}-$ $H), 8.02(\mathrm{~s}, 1 \mathrm{H}, \mathrm{NH}) ;{ }^{13} \mathrm{C} \mathrm{NMR}\left(100 \mathrm{MHz}, \mathrm{CDCl}_{3}\right) \delta 20.8,22.5,32.5$ and $32.6\left(2 \mathrm{~d}, J_{(\mathrm{P}-\mathrm{C})} \sim 4.4 \mathrm{~Hz}\right.$, $\left.C \mathrm{Me}_{2}\right), 52.7\left(\mathrm{t}, J_{(\mathrm{P}-\mathrm{C})}=136.2 \mathrm{~Hz}, \mathrm{P}-\mathrm{C}-\mathrm{P}\right), 78.1,115.5,118.6,119.8,121.2,122.0,125.1,126.0$, 127.5, 128.8, 129.0, 131.0, 132.6, 134.8, 138.6; ${ }^{31} \mathrm{P}$ NMR (162 MHz, $\left.\mathrm{CDCl}_{3}\right) \delta 8.0$ (s). Anal. Calcd for $\mathrm{C}_{27} \mathrm{H}_{31} \mathrm{NO}_{6} \mathrm{P}_{2}:$ C, 61.48; H, 5.92; N, 2.66. Found: C, 61.42; H, 5.96; N, 2.73\%.

[9-(Diethoxy-phosphoryl)-9,10-dihydro-acridin-9-yl]-phosphonic acid diethyl ester (17). Yield [by NMR; isolated] 60\%, $0.60 \mathrm{~g}(25 \%)$; mp 214-216 ${ }^{\circ} \mathrm{C}$; white solid; IR ( $\left.\mathrm{KBr}, \mathrm{cm}^{-1}\right) 3266,2963$, 2894, 1613, 1530, 1491, 1344, 1250, 743; ${ }^{1} \mathrm{H}$ NMR (400 MHz, $\left.\mathrm{CDCl}_{3}\right) \delta 1.16$ (t, $12 \mathrm{H}, J=7.2 \mathrm{~Hz}$, $\left.\mathrm{OCH}_{2} \mathrm{CH}_{3}\right), 4.00-4.10\left(\mathrm{~m}, 8 \mathrm{H},-\mathrm{OCH}_{2} \mathrm{CH}_{3}\right), 6.12(\mathrm{~s}, 1 \mathrm{H},-\mathrm{NH}), 6.47(\mathrm{~d}, 2 \mathrm{H}, J=7.6 \mathrm{~Hz}, \mathrm{Ar}-\mathrm{H})$, $6.78(\sim \mathrm{t}, 2 \mathrm{H}, J=7.6 \mathrm{~Hz}, \mathrm{Ar}-H), 7.07$ ( t, $2 \mathrm{H}, J \sim 7.6 \mathrm{~Hz}, \mathrm{Ar}-H), 8.16(\mathrm{~d}, 2 \mathrm{H}, J=7.6, \operatorname{Ar}-H) ;{ }^{13} \mathrm{C}$ $\operatorname{NMR}\left(100 \mathrm{MHz}, \mathrm{CDCl}_{3}\right) \delta 16.2$ and $16.3\left(2 \mathrm{~d}, J_{(\mathrm{P}-\mathrm{C})} \sim 2.7 \mathrm{~Hz}, \mathrm{OCH}_{2} \mathrm{CH}_{3}\right), 50.5\left(\mathrm{t}, J_{(\mathrm{P}-\mathrm{C})}=132.5 \mathrm{~Hz}\right.$, P-C-P), 63.8 and $63.9\left(2 \mathrm{~d}, J_{(\mathrm{P}-\mathrm{C})} \sim 3.5 \mathrm{~Hz}, \mathrm{OCH}_{2} \mathrm{CH}_{3}\right), 112.0\left(\mathrm{t}, J_{(\mathrm{P}-\mathrm{C})}=8.2 \mathrm{~Hz}\right), 114.0,118.8$, 129.0, 133.3, $138.7\left(\mathrm{t}, J_{(\mathrm{P}-\mathrm{C})}=5.5 \mathrm{~Hz}\right) ;{ }^{31} \mathrm{P} \mathrm{NMR}\left(162 \mathrm{MHz}, \mathrm{CDCl}_{3}\right) \delta 15.9(\mathrm{~s}) ; \mathrm{LC}-\mathrm{MS} \mathrm{m} / \mathrm{z}=453$ $[\mathrm{M}]^{+}$. Anal. Calcd for $\mathrm{C}_{21} \mathrm{H}_{29} \mathrm{NO}_{6} \mathrm{P}_{2}$ : C, 55.63; H, 6.45; N, 3.09. Found: C, 55.71; H, 6.41; N, $3.12 \%$. This compound was crystallized from EtOAc-hexane $(4+1 \mathrm{~mL})$. X-Ray structural analysis was performed on this sample (right diagram in Figure 1). This compound has been mentioned in the literature before. ${ }^{15}$

\section{(c) Synthesis of 9-(5,5-dimethyl-2-oxo-2 $\lambda^{5}$-[1,3,2]dioxaphosphinan-2-yl)-acridine (18)}

A mixture of 9-chloro-2-methyl-acridine $(1.14 \mathrm{~g}, 5 \mathrm{mmol})$ and the phosphite 10 (0.60 g, $4 \mathrm{mmol})$ were heated at $90{ }^{\circ} \mathrm{C}$ under nitrogen for $4 \mathrm{~h}$. Ethyl acetate $(20 \mathrm{~mL})$ was added to the reaction mixture followed by saturated sodium bicarbonate solution. The organic layer was separated and the aqueous layer extracted thrice $(3 \times 10 \mathrm{~mL})$ with ethyl acetate. The combined organic layer was washed with water $(5 \times 20 \mathrm{~mL})$, brine solution, dried over anhyd. $\mathrm{Na}_{2} \mathrm{SO}_{4}$, and concentrated to yield a brown gummy solid. Pure 9-(5,5-dimethyl-2-oxo-2- $\lambda^{5}-[1,3,2]$ dioxaphosphinan-2-yl)-acridine (18) 
was isolated by column chromatography (ethyl acetate: hexane, 2:3) as a pale yellow solid. Yield [by NMR; isolated] 20\%, $0.17 \mathrm{~g}(10 \%) ; \mathrm{mp} \mathrm{182-184}{ }^{\circ} \mathrm{C}$; IR $\left(\mathrm{KBr}, \mathrm{cm}^{-1}\right) 2974,2924,1634,1474$, 1262, 1051, 1009; ${ }^{1} \mathrm{H} \mathrm{NMR}\left(400 \mathrm{MHz}, \mathrm{CDCl}_{3}\right) \delta 0.67$ and $1.44\left(2 \mathrm{~s}, 6 \mathrm{H}, 2 \mathrm{CH}_{3}\right), 2.63(\mathrm{~s}, 3 \mathrm{H}$, $\left.\mathrm{CH}_{3}\right), 3.59-3.63\left(\mathrm{~m}, 2 \mathrm{H}, \mathrm{OCH}_{2}\right), 3.99-4.07\left(\mathrm{~m}, 2 \mathrm{H}, \mathrm{OCH}_{2}\right), 7.63-7.83(\mathrm{~m}, 3 \mathrm{H}, \mathrm{Ar}-\mathrm{H}), 8.22(\mathrm{~d}, 1 \mathrm{H}$, $J=8.8 \mathrm{~Hz}, \mathrm{Ar}-H), 8.30$ (d, $1 \mathrm{H}, J=7.6 \mathrm{~Hz}, \mathrm{Ar}-H), 8.57$ (s, $1 \mathrm{H}, \mathrm{Ar}-H) ; 8.80$ (d, $1 \mathrm{H}, J=8.8 \mathrm{~Hz}, \mathrm{Ar}-$ $H) ;{ }^{13} \mathrm{C} \mathrm{NMR}\left(100 \mathrm{MHz}, \mathrm{CDCl}_{3}\right) \delta 20.4,22.2,22.5,32.1\left(\mathrm{~d}, J_{(\mathrm{P}-\mathrm{C})}=6.0 \mathrm{~Hz}, \mathrm{CMe}_{2}\right), 76.8,76.9$, $124.3\left(\mathrm{~d}, J_{(\mathrm{P}-\mathrm{C})}=5.8 \mathrm{~Hz}\right), 126.0\left(\mathrm{~d}, J_{(\mathrm{P}-\mathrm{C})}=5.7 \mathrm{~Hz}\right), 126.8,127.1,127.2,127.8,129.5,130.2,130.6$, 133.0, 138.2 [the doublet due to ${ }^{1} J_{(\mathrm{P}-\mathrm{C})}$ was not clear]; ${ }^{31} \mathrm{P} \mathrm{NMR}\left(162 \mathrm{MHz}, \mathrm{CDCl}_{3}\right) \delta 10.6$ (s); LCMS $m / z=342[\mathrm{M}+1]^{+}$. Anal. Calcd for $\mathrm{C}_{19} \mathrm{H}_{20} \mathrm{NO}_{3} \mathrm{P}: \mathrm{C}, 66.85 ; \mathrm{H}, 5.91 ; \mathrm{N}, 4.10$. Found: C, 66.81; $\mathrm{H}, 5.86 ; \mathrm{N}, 4.18 \%$.

\section{X-ray crystallography}

X-Ray data were collected on a Bruker AXS SMART diffractometer using Mo-K $\alpha(\lambda=0.71073 \AA)$ radiation. The structures were solved and refined by standard methods. ${ }^{23}$ All non-hydrogen atoms were refined anisotropically. Crystal data have been deposited in the Cambridge database.

\section{Crystal data for compounds (11) $\mathrm{CH}_{3} \mathrm{CN}$, (14) $\mathrm{OC}_{4} \mathrm{H}_{8}$ and (17)}

(11) $\mathrm{CH}_{3} \mathrm{CN}: \mathrm{C}_{71} \mathrm{H}_{90} \mathrm{~N}_{4} \mathrm{O}_{18} \mathrm{P}_{6}, \mathrm{M}=1473.29$, Trigonal, Space group $\mathrm{R}-3$, a $=21.029(3), \mathrm{b}=$ 21.029(3), $\mathrm{c}=28.896(7) \AA, \alpha=\beta=90.0^{\circ}, \gamma=120.0^{\circ}, \mathrm{V}=11067$ (3) $\AA^{3}, \mathrm{Z}=6, \mu=0.217 \mathrm{~mm}^{-1}$, data/restraints/parameters: 4237/0/311, R indices $(\mathrm{I}>2 \sigma(\mathrm{I})$ ): R1 $=0.0785$, wR2 (all data) $=0.1638$. Max./min. residual electron density $\left(\mathrm{e} \AA^{-3}\right) 0.628 /-0.243$. CCDC No. 727444.

(14) $\mathrm{C}_{4} \mathrm{H}_{8} \mathrm{O}: \mathrm{C}_{27} \mathrm{H}_{36} \mathrm{ON}_{2} \mathrm{O}_{9} \mathrm{P}_{2}, \mathrm{M}=594.52$, Monoclinic, Space group $\mathrm{P} 2(1) / \mathrm{c}, \mathrm{a}=15.240(5), \mathrm{b}=$ 10.453(3), $\mathrm{c}=21.548(7) \AA, \beta=100.909(5)^{\circ}, \mathrm{V}=3370.6(19) \AA^{3}, \mathrm{Z}=4, \mu=0.176 \mathrm{~mm}^{-1}$, data/restraints/parameters: 5928/0/410, R indices $(\mathrm{I}>2 \sigma(\mathrm{I}))$ : R1 $=0.1013$, wR2 (all data) $=0.2839$. Max./min. residual electron density $\left(\mathrm{e}^{-3}\right) 0.918 /-0.417$. CCDC No. 727445.

17: $\mathrm{C}_{21} \mathrm{H}_{29} \mathrm{NO}_{6} \mathrm{P}_{2}, \mathrm{M}=453.39$, Monoclinic, Space group P2(1), a = 8.096(6), $\mathrm{b}=15.300(11), \mathrm{c}=$ 19.538(7) $\AA, \beta=96.211(13)^{\circ}, \mathrm{V}=1174.5(15) \AA^{3}, \mathrm{Z}=2, \mu=0.220 \mathrm{~mm}^{-1}$, Flack parameter 0.01(14), data/restraints/parameters: 4011/1/279, R indices $(\mathrm{I}>2 \sigma(\mathrm{I}))$ : R1 $=0.0681$, wR2 (all data) $=0.1252$. Max./min. residual electron density $\left(\mathrm{e} \AA^{-3}\right) 0.242 /-0.204$. CCDC No. 727446 .

\section{Acknowledgements}

We acknowledge financial and equipment support from the Department of Science and Technology (DST), Council of Scientific and Industrial Research (CSIR), and University Grants Commission (UGC) [all in New Delhi]. V.S. thanks the CSIR for a fellowship.

\section{References}

1. Review pertaining to acridine chemistry: Chiron, J.; Galy, J.-P. Synthesis 2004, 313. 
2. (a) Kuruvilla, E.; Andaman, P. C.; Schuster, G. B.; Ramaiah, D. Org. Lett. 2008, 10, 4295. (b) Benniston, A. C.; Rewinska, D. B. Org. Biomol. Chem. 2006 4, 3886. (c) Mizuki, K.; Sakakibara, Y.; Ueyama, H.; Nojima, T.; Waki, M.; Takenaka, S. Org. Biomol. Chem. 2005, 3, 578.

3. Motoyoshiya, J.; Ikeda, T.; Tsuboi, S.; Kusaura, T.; Takeuchi, Y.; Hayashi, S.; Yoshioka, S.; Takaguchi, Y.; Aoyama, H. J. Org. Chem. 2003, 68, 5950.

4. (a) Cheng, M.-K.; Modi, C.; Cookson, J. C.; Hutchinson, I.; Heald, R. A.; McCarroll, A. J.; Missailidis, S.; Tanious, F.; Wilson, W. D.; Mergny, J.-L.; Laughton, C. A.; Stevens, M. F. G. J. Med. Chem. 2008, 51, 963. (b) Sánchez, I.; Reches, R.; Caignard, D. H.; Renard, P.; Pujol, M. D. Eur. J. Med. Chem. 2006, 41, 340. (c) Bacherikov, V. A.; Chang, J.-Y.; Lin, Y.-W.; Chen, C.-H.; Pan, W.-Y.: Dong, H.; Lee, R.-Z.; Chou, T.-C.; Su, T.-L. Bioorg. Med. Chem. 2005, $13,6513$.

5. Wainwright, M. J. Antimicrob. Chemother. 2001, 47, 1.

6. Hamy, F.; Brondani, V.; Flörsheimer, A.; Stark, W.; Blommers, M. J. J.; Limkait, T. Biochemistry 1998, 37, 5086.

7. (a) Jones, M.; Mercer, A E.; Stocks, P. A.; Pensée, L. J. I. L.; Cosstick, R.; Park, B. K.; Kennedy, M. E.; Piantanida, I.; Ward, S. A.; Davies, J.; Bray, P. G.; Rawe, S. L.; Baird, J.; Charidza, T.; Janneh, O.; O’Neill, P. M. Bioorg. Med. Chem. Lett. 2009, 19, 2033. (b) Zsila, F.; Visy, J.; Mady, G.; Fitos, I. Bioorg. Med. Chem. 2008, 16, 3759.

8. (a) Kuzuya, A.; Mizoguchi, R.; Sasayama, T.; Zhou, J.-M.; Komiyama, M. J. Am. Chem. Soc. 2004, 126, 1430. (b) Joseph, J.; Eldho, N. V.; Ramaiah, D. Chem. Eur. J. 2003, 9, 5926 (c) Antonini, I.; Polucci, P.; Magnano, A.; Martelli, S. J. Med. Chem. 2001, 44, 3329.

9. (a) Giorgio, C. D.; Shimi, K.; Boyer, G.; Delmas, F.; Galy, J.-P. Eur. J. Med. Chem. 2007, 42, 1277. (b) Carole, D. G.; Michel, D. M.; Julien, C.; Florence, D.; Anna, N.; Severine, J.; Gerard, D.; Pierrea, T. -D.; Galy, J.-P. Bioorg. Med. Chem. 2005, 13, 5560. (c) Giorgio, C. D.; Delmas, F.; Filloux, N.; Robin, M.; Seferian, L; Azas, N.; Gasquet, M.; Costa, M.; David, P. T.; Galy, J.P. Antimicrob. Agents Chemother. 2003, 47, 174.

10. (a) Praveen Kumar, K.; Muthiah, C.; Kumaraswamy, S.; Kumara Swamy, K. C. Tetrahedron Lett. 2001, 42, 3219. (b) Senthil Kumar, K.; Kumara Swamy, K.C. J. Organomet. Chem. 2001, 637, 616. (c) Muthiah, C.; Senthil Kumar, K.; Vittal, J. J.; Kumara Swamy, K. C. Synlett 2002, 11, 1787. (d) Chakravarty, M.; Srinivas, B.; Muthiah, C.; Kumara Swamy, K. C. Synthesis 2003, 2368. (e) Kumara Swamy, K. C.; Balaraman, E.; Satish Kumar, N. Tetrahedron 2006, 62, 10152. (f) Chakravarty, M.; Kumara Swamy, K. C. J. Org. Chem. 2006, 71, 9128. (g) Kumara Swamy, K. C.; Srinivas, V.; Pavan Kumar, K. V. P.; Praveen Kumar, K. Synthesis 2007, 893.

11. (a) Baillie, C.; Xiao, J. Curr. Org. Chem. 2003, 7, 477. (b) Tanaka, M. Top. Curr. Chem. 2004, 232, 25. (c) Montchamp, J.-L. J. Organomet. Chem. 2005, 690, 2388. (d) Troev, K. D. Chemistry and Application of H-Phosphonates, Elsevier: Amsterdam, 2006.

12. Russel, R. G. G.; Rogers, M. J. Bone 1999, 25, 97.

13. Rouband, G.; Faure, R.; Galy, J.-P. Magn. Reson. Chem. 2003, 41, 549.

14. Feng, S.; Panetta, C. A.; Graves, D. E. J. Org. Chem. 2001, 66, 612. 
15. Also see: (a) Kosolapoff, G. M. J. Am. Chem. Soc. 1947, 69, 1002. (b) Redmore, D. J. Org. Chem. 1969, 34, 1420.

16. (a) Chen, Y.-L.; Chen, I.-L.; Lu, C.-M.; Tzeng, C.-C.; Tsao, L.-T.; Wang, J.-P. Bioorg. Med. Chem. 2003, 11, 3921.

17. Chakravarty, M.; Bhuvan Kumar, N. N.; Sajna, K. V.; Kumara Swamy, K. C. Eur. J. Org. Chem. 2008, 4500.

18. Kumara Swamy, K. C.; Kumaraswamy, S.; Kommana, P. J. Am. Chem. Soc. 2001, 123, 12642.

19. Roy, K. R.; Arunasree, K. M.; Reddy, N. P.; Dheeraj, B.; Reddy, G. V.; Reddanna, P. Biotechnol. Appl. Biochem. 2007, 47, 159.

20. Perrin, D. D.; Armarego, W. L. F.; Perrin, D. R. Purification of Laboratory Chemicals; Pergamon: Oxford, UK, 1986.

21. Wolf, C.; Liu, S.; Mei, X.; August, A. T.; Casimir, M. D. J. Org. Chem. 2006, 71, 3270.

22. Muthiah, C.; Praveen Kumar, K.; Aruna Mani, C.; Kumara Swamy, K. C. J. Org. Chem. 2000, $65,3733$.

23. (a) Sheldrick, G. M. SHELX-97- A Program for Crystal Structure Solution and Refinement, University of Göttingen, 1997. (b) Sheldrick, G. M. SADABS, Siemens Area Detector Absorption Correction, University of Göttingen, Germany, 1996. (c) Sheldrick, G. M. SHELXTLNT Crystal Structure Analysis Package, Bruker AXS, Analytical X-ray System, WI, USA, 1999, Version 5.10. 